\title{
Lethal outcome of catastrophic antiphospholipid syndrome despite intensive triple therapy
}

\author{
Carlos Anjo*1, Philip Fortuna ${ }^{2}$, Luís Reis ${ }^{2}$, Luís Bento² \\ ${ }^{1}$ Serviço de Medicina Interna III, Hospital Pulido Valente, Centro Hospitalar Lisboa Norte, Lisbon, Portugal \\ ${ }^{2}$ Unidade de Urgência Médica, Hospital de São José, Centro Hospitalar Lisboa Central, Lisbon, Portugal
}

Received: December 18, 2018

Accepted: January 16, 2019

Online Published: January 22, 2019

DOI: $10.5430 /$ crim.v6n $1 \mathrm{p} 13$

URL: https://doi.org/10.5430/crim.v6n1p13

\begin{abstract}
Catastrophic antiphospholipid syndrome (CAPS), an infrequent autoimmune disease, manifests as a widespread thrombotic disease with multiorgan failure with a $37 \%$ mortality rate. We report 39-year-old Caucasian women, medical history of Systemic Lupus Erythematous (SLE) complicated with APS and proliferative glomerulonephritis under dialysis. Patient was admitted at the ICU with septic shock with thoracotomy dehiscence origin. During the ICU stay, extensive lower limbs ulcers with polymorphonuclear infiltrate and steatonecrosis areas in adipose and muscular tissue. Lab studies: anti- $\beta_{2}$-glycoprotein-I antibody was detected. As probable CAPS diagnosis was made, triple therapy with methylprednisolone, immunoglobulin and anticoagulation immediately was started. Serious vasoplegic shock occurs with failure in vasopressor support increment and the patient died. The authors report an APS with rapid progression to catastrophic stage, rare complication that occurs only in $1 \%$ of catastrophic APS. In CAPS treatment, a statistical association between triple therapy and the decrease of mortality rate of patients with CAPS was observed, when compared with other combinations or with none of the treatments included in the triple therapy. Although the case had a lethal outcome, the authors emphasize the use of triple therapy in CAPS and the challenge of treating patients with CAPS and infection where the balance between the need for anticoagulation, aggressive immunosuppression and infection control is difficult to manage.
\end{abstract}

Key Words: Catastrophic antiphospholipid syndrome, Triple therapy, Intensive care, Systemic lupus erythematosus

\section{INTRODUCTION}

Antiphospholipid syndrome (APS) is an autoimmune disorder that manifests clinically as recurrent venous or arterial thrombosis and/or fetal loss, in the presence of antiphospholipids antibodies. It has an incidence of 5 new cases per 100,000 persons / year and a prevalence of 40-50 cases per 10,000 persons. ${ }^{[1]}$ The prevalence of the catastrophic antiphospholipid syndrome (CAPS) is scarce, less than $1 \%$ of all cases of APS ${ }^{[2]}$ it manifests clinically as disseminated thrombotic disease with multiple organ disfunction and a
$37 \%$ mortality rate. ${ }^{[3]}$

\section{Case representation}

We present 39 years old women, with known history of systemic lupus erythematous (SLE), APS, proliferative glomerulonephritis and terminal renal disease on dialysis with previous transplant rejection in 2011. Medicated with acetylsalicylic acid $100 \mathrm{mg}$ id, enoxaparin $80 \mathrm{mg} 2 \mathrm{id}$, hydroxychloroquine $400 \mathrm{mg}$ id, mycophenolate mofetil $500 \mathrm{mg} 2 \mathrm{id}$ and rituximab $500 \mathrm{mg}$ since 2013 (last administration 4 months

\footnotetext{
*Correspondence: Carlos Anjo; Email: carloslccanjo@gmail.com; Address: Hospital Pulido Valente, CHLN, Alameda das linhas de Torres, 117, 1600, Lisbon, Portugal.
}

Published by Sciedu Press 
before).

Admitted at the hospital by acute myocardial infarction, submitted to angioplasty and CABG (coronary artery bypass graft). A complication was detected after the procedure with thoracotomy wound dehiscence and suture infection. Several cycles of antibiotic were performed to control the infection, she was discharge under vacuum-assisted closure therapy. After one month, the patient was admitted again at the emergency department, with fever, prostration and increased acute phase reactants (C-reactive protein 1,084 $\mathrm{mg} / \mathrm{L}$ ) in blood tests. A thoracic CT scan revealed osteonecrosis and osteomyelitis signs. The patient was admitted at the intensive care unit (ICU) with septic shock from surgical wound, empiric antibiotherapy was started with meropenem and vancomycin. Vasopressor support with noradrenalin was required, maximal dose $13 \mathrm{mcg} / \mathrm{min}$. P. aeruginosa and E. Coli bacteraemia was detected and a switch to piperacillin/tazobactam was performed based in antibiotic susceptibility test. A 21 days cycle of meropenem was made based in intra-op exudate with K. pneumoniae ESBL. During hospitalization period, P. aeruginosa (carbapenem-resistant) and E. Faecalis was collected from lower limbs exudate, linezolid and ceftazidime was initiated.

A polymorphonuclear inflammatory infiltrate, with steatonecrosis areas in adipose and muscular tissue, was found on histological evaluation from inferior limbs wounds. The patient started with symptoms of sensorial clouding and conscience state fluctuation, from uremic origin. CT scan and lumbar puncture do not reveal changes. Mechanical ventilation was required after cutaneous wounds debridement, extubation was successful achieved on the fourth postoperative day.

Immunology revealed positive anti- $\beta_{2}$-glycoprotein-I antibody, remaining antiphospholipid antibodies were negative. Based on a probable diagnosis of CAPS, triple therapy was started with high doses intravenous corticotherapy (1 g/day methylprednisolone for three days), intravenous immunoglobulin (for 5 days), and anticoagulation with enoxaparin (started at admission).

A large haematoma in left thigh appeared as complication, a blood transfusion was made as a result of a $3 \mathrm{~g} / \mathrm{L}$ drop in haemoglobin. A colonoscopy was performed, as a result of gastro intestinal haemorrhage, multiples profound ulcers were observed across the colon. IgM serology, and polymerase chain reaction test, were both negative for cytomegalovirus. A sigmoid colon biopsy revealed vascular and multifocal ischemic ulcerations, associated with amyloid deposits.

Iliac artery thromboembolectomy was performed, as a result of acute ischemia in right lower limb, caused by an arterial thrombus. A new rectorrhagia episode had occur in postoperative period, with $3 \mathrm{~g} / \mathrm{L}$ haemoglobin loss, an abundant blood transfusion was performed. A severe vasoplegic shock with vasopressor failure occurred and unfortunately the patient died.

Table 1. Classification criteria for catastrophic antiphospholipid syndrome ${ }^{[5]}$

1. Evidence of involvement of three or more organs, systems and/or tissues
2. Development of manifestations simultaneously or in less than a week
3. Confirmation by histopathology of small-vessel occlusion*
4. Laboratory confirmation of the presence of antiphospholipid antibodies $\dagger$
Definite catastrophic antiphospholipid syndrome
- $\quad$ All four criteria present
- $\quad$ Probable catastrophic antiphospholipid syndrome
- $\quad$ All four criteria, except only two organs, systems, and/or tissues involved
- $\quad$ All four criteria, except for the absence of laboratory confirmation of antiphospholipid antibodies
- $\quad$ Criteria 1,2 , and 4
anticoagulation 1,3 , and 4 , with the development of a third event more than 1 week but within 1 month of presentation, despite

Note. *Vasculitis may coexist, but significant thrombosis must be present as well. †"Positive aPL” twice 12 weeks apart.

\section{Discussion}

According to international consensus for CAPS diagnosis (see Table 1$),{ }^{[5]}$ three of four criteria were confirmed: involvement of two organs, systems or tissues - microthrombus in sigmoid colon and acute ischemia in right lower limb with the presence of thrombus confirmed by doppler; confirmation by histopathology of small vessel occlusion in at least one organ or tissue-sigmoid biopsy with microthrombus; and history of APS-vascular thrombosis confirmed by imaging and anticardiolipin IgG antibody measured in two 
occasion, 1 year apart. A probable CAPS was assumed according the international consensus criteria. With respect to differential diagnosis, thrombotic microangiopathies were excluded because no renal affection was observed. Heparin induced thrombocytopenia were also excluded because heparin-PF4 antibodies were negative and the patient had several exposures to heparin in the past. Disseminated intravascular coagulation diagnose was also excluded because no fibrinolysis was observed, as d-dimer levels were normal. The authors discuss a patient with APS secondary to SLE, CAPS it's a rare situation that only occurs in $1 \%$ of APS patients. ${ }^{[2]}$ In this case report, the trigger was the infection, it can trigger CAPS in $22 \%$ of cases. ${ }^{[2]}$

An unsuccessful attempt to control CAPS progression was

\section{REFERENCES}

[1] Cervera R. Antiphospholipid syndrome. Thromb Res. 2017; 151 Suppl 1: S43-S47. https ://doi .org/10.1016/S0049-3848(17 ) 30066-X

[2] Espinosa G, Rodríguez-Pintó I, Cervera R. Catastrophic antiphospholipid syndrome: an update. Panminerva Med. 2017; 59(3): 254-268.

[3] Cervera R. The diagnosis and clinical management of the catastrophic antiphospholipid syndrome: a comprehensive review. J Autoimmun. 2018; 92: 1-11. https://doi.org/10.1016/j.jaut.2018.05 .007 performed with triple therapy-immunoglobulin, methylprednisolone and anticoagulation. Triple therapy use in CAPS is described in several studies, Rodríguez-Pintó et al study shows a statistical association between triple therapy and the decrease of mortality rate in patients with CAPS, when compared with other combinations or with none of the treatments included in triple therapy. ${ }^{[4]}$ Although the case had a lethal outcome, the authors emphasize the use of triple therapy in CAPS and the challenge of treating patients with CAPS and infection where the balance between the need for anticoagulation, aggressive immunosuppression and infection control is difficult to manage.

\section{Conflicts of InTEREST Disclosure}

The authors have declared no conflicts of interest.
[4] Rodríguez-Pintó I, Espinosa G, Erkan D, et al. CAPS Registry Project Group (2018) The effect of triple therapy on the mortality of catastrophic antiphospholipid syndrome patients. Rheumatology. 2018; 57(7): 1264-1270 . https ://doi .org/10.1093/rheumato logy/key082

[5] Asherson RA, Cervera R, de Groot PG, et al. Catastrophic antiphospholipid syndrome: international consensus statement on classification criteria and treatment guidelines. Lupus. 2003; 12(7): 530-4. https://doi.org/10.1191/0961203303lu394oa 